\title{
The Prevalence of Premature Ovarian Failure among Subfertile Patient in Albayda Fertility Centre/A Public Center in Libya
}

\author{
Agzail Saad Elhddad M.D*
}

Senior Consultant at Albayda Fertility Teaching Centre; Assistant Professor, Department of Obstetrics \& Gynecology, Faculty of Medicine, Omar ElMukhtar University- Albayda/Libya

DOI: $\underline{10.36348 / \text { sijap.2020.v03i05.002 }}$

| Received: 12.05.2020 | Accepted: 19.05.2020 | Published: 30.05.2020

*Corresponding author: Agzail Saad Elhddad

Abstract

Background: Premature ovarian failure (PMOF) associated with increased risk of the general and mental health of the affected women, however, lower fertility or even infertility are the most disturbing symptoms to every woman with PMOF. Therefore, this study was conducted to assess the prevalence of PMOF among infertile women as this may reflect the real magnitude of the problem in the whole population. Methodology: women aged 40 years or less with infertility and menstrual irregularity (oligomenorrhea or amenorrhea) and with an elevated FSH level on two occasions more than 4 weeks apart were recruited for this study. The participants were attending Albayda Fertility Centre a public centre in Libya, between August 2013 and January 2020. The results: the recorded prevalence of PMOF among infertile subjects in this study was $2.8 \%$. 34\% of the affected women were aged 35 years and below. Only 2 (3.45\%) of them got spontaneous pregnant, but none ended in live-birth. The median age at presentation was 36 years. The median infertility duration was 4.5 years and the median duration of menstrual irregularity was 7 years. The menstrual irregularity predated infertility by a median of 2.5 years. Conclusion: the prevalence of PMOF in this study was $2.8 \%$ indicating a higher prevalence of PMOF among infertile women than previously reported prevalence in the general population. This study also confirms the low reproductive potential of these women.

Keywords: PMOF, menopause, FSH, AMH, oligomenorrhea, amenorrhea, infertility.

Copyright @ 2020: This is an open-access article distributed under the terms of the Creative Commons Attribution license which permits unrestricted use, distribution, and reproduction in any medium for non-commercial use (NonCommercial, or CC-BY-NC) provided the original author and source are credited.

\section{INTRODUCTION}

Premature ovarian failure (PMOF) is a clinical disorder manifested with menstrual disturbance (oligomenorrhea or amenorrhea) in the female population at or under the age of 40 years [1]. PMOF diagnosed by elevated serum gonadotropins, in particular FSH (FSH level > 25 IU/l on two occasions > 4 weeks apart) [1, 2]. The current estimate of the prevalence of PMOF is very variable and ranged between 0.9 and $2 \%$ in most studies [1, 3-5].

The possible potential mechanisms leading to PMOF could be; a congenital low count of primordial follicles or their accelerated atresia, or inability to recruit these follicles [6]. The vast majority of spontaneous PMOF cases are idiopathic [7]. The known causes of PMOF are wide-ranging and could be due to pelvic or ovarian surgery, chemotherapy, radiotherapy, genetic causes, metabolic and storage disorders, infections, chromosomal anomalies, or autoimmune diseases [8].
As they ovulate extremely rarely, low fertility or even infertility are the most disturbing symptoms to every woman with PMOF, and only 5\% of them got pregnant spontaneously [7]. PMOF not only affecting the woman's reproductive potential, but also associated with increased risk of hypoestrogenism-related problems such as heart disease, osteoporosis, and early mortality [9-12]. These women may also suffer from other autoimmune problems such as autoimmune hypothyroidism or adrenal insufficiency [13, 14], in addition to other co-morbidities such as depression and anxiety $[15,16]$. Therefore, PMOF could be considered as a substantial health issue, not only for the affected person, but also for her family and the general public health.

As there are no data concerning the actual prevalence of PMOF available to date, among the Libyan population, we aimed to assess the prevalence of PMOF among infertile women attending Albayda Fertility center (afc.med.ly), a public teaching center in Libya as this may reflect the real prevalence of this 
problem in the whole Libyan population. In an attempt to reduce the incidence of PMOF in the future by modifying potential risk factors to preserve fertility as well as to reduce the associated long-term health consequences.

Future work: to include patients with PMOF attending other infertility centers in Libya in one study to find out the real magnitude and the possible causes of the problem, especially the preventable risk factors, this could help to limit the consequences of the PMOF.

\section{METHODOLOGY}

As shown in the flow chart 1, women aged between 20 and 40 years with infertility and menstrual irregularity (oligomenorrhea or amenorrhea) were recruited for this study from the infertility clinic at Albayda Fertility Centre (afc.med.ly) in Albayda, Libya, from August 2013 to January 2020. Ethical approval was obtained from the local Ethics Committee and the couples were counseled to participate in the study.

A pre-prepared proforma was used to collect the personal data, demographic data (age, BMI, blood pressure, and area of residence; urban or suburban). Past obstetric history, as well as a history of malignant and chronic diseases, surgical, gynecological history and family history of the same problem (PMOF), were collected. Educational and occupational history, lifestyle data (smoking, alcohol consumption) have been registered.

After that, the work-up consisting of baseline assessment of FSH, LH and $\mathrm{E}_{2}$ levels (day 2 or 3 of the menstrual cycle; either natural or drug-induced) and transvaginal ultrasound to assess the antral follicle count (AFC) was performed. As reported before, both $\mathrm{FSH}$ and AMH levels should be measured to assess the ovarian reserve $[17,18]$. Therefore, all the subjects, then underwent a test of AMH level (blood samples were taken for AMH levels on any day of the menstrual cycle). Serum prolactin and TSH were also estimated. Four weeks later, a second blood sample was taken from all the participants with a high FSH level to reassess the FSH level to satisfy the diagnostic criteria of $\mathrm{PMOF}$ as recommended $[1,2]$.

The primary outcome measure in this study was to assess the prevalence of PMOF among infertile women and the secondary outcome to assess their reproductive outcome.

Statistical analysis: data was entered and analyzed using SPSS software (version 25). The overall prevalence of PMOF and the prevalence of PMOF within different age groups as well as the reproductive outcome of the participants were presented with absolute numbers and percentages. The number and percentages were also computed for qualitative variables; type of infertility (primary and secondary) and area of residence (urban and suburban). The quantitative variables, including age, duration of menstrual irregularities and infertility, basal FSH, LH and estradiol levels, AMH level, body mass index (BMI) were presented by medians and range.

\section{RESULTS}

2842 couples were seeking infertility treatment attending Albayda Fertility Centre (afc.med.ly) between August 2013 and January 2020. 2072 women included in the study after excluding cases with pure male factor (650 couples) and those who withdraw from the study for social reasons or because of the lack of follow up (120 couples). 58 women satisfied the criteria of PMOF with an overall PMOF prevalence of $2.8 \%$ among the infertile participants attending our center during the study period. Table 1 shows the patient's distribution according to the age group: 26 patients (35\%) were aged between 20 and 35 years and $55 \% \%$ of women with PMOF aged between 36 and 40 years. Out of these 58 cases, 2 of them got spontaneous pregnancy $(3.45 \%)$, one of them ended in first-trimester miscarriage $(1.7 \%)$ and the other case ended in an ectopic pregnancy $(1.7 \%)$ and was undergone salpingectomy.

The baseline characteristics of patients presented in Table-2. The median age at presentation was 36 years (range from 19 to 40 years). All patients were presented with either primary or secondary infertility and the median infertility duration was 4.5 years (ranged from 1 to 14 years). $60.5 \%$ of women were presented with oligomenorrhea whereas amenorrhea (either primary or secondary) was the main complaint of 23 women. The median duration of menstrual irregularity (oligomenorrhea and amenorrhea) was 7 years and ranged between 1 and 16 years. The menstrual irregularity predated infertility by a median of 2.5 years.

The median BMI of the included patients was $30 \mathrm{~kg} / \mathrm{m}^{2}$. No patient gave a history of cigarette smoking or alcohol drinking also none of them gave a history of other concomitant diseases (allergies, thyroid diseases, type 2 diabetes mellitus, hypertension, or breast cancer). There was no significant difference concerning the residence (urban or suburban areas). Most of the patients were either teachers or housewife and most of them were with a secondary school level or University education.

Concerning the past obstetric history; 9 women got spontaneous pregnancy (4 of them gave live-birth, 4 ended in miscarriage and one ended in ectopic pregnancy). 6 couples had trails of intrauterine insemination whereas 11 couples had intracytoplasmic sperm injection trails (ICSI) however all these trails failed. 
Only two patients $(3.45 \%)$ gave a family history of the same problem. Three cases gave a history of ovarian cystectomy, two had unilateral oophorectomy and only one had ovarian drilling. 14 cases gave a history of lower abdominal or pelvic surgery; most of them were either appendicectomy or cesarean section. None of the patients used oral contraceptives or received chemotherapy or radiotherapy.
As shown in Table- 3 the median and range of the first measured FSH level was 31 (28-114) IU/l and the result of the second FSH level taken after 4 weeks was 46 (30-316) IU/l. The median level of LH was 22 (29-62) IU/l and that of estradiol was 15 (1-58) ng/ml. The median and range of AMH level was $0.13(0.01-$ 1.2) $\mathrm{ng} / \mathrm{ml}$ and the median and range of the AFC was 1 $(0-5)$. The serum levels of TSH and prolactin were within the normal range for all the included participants.

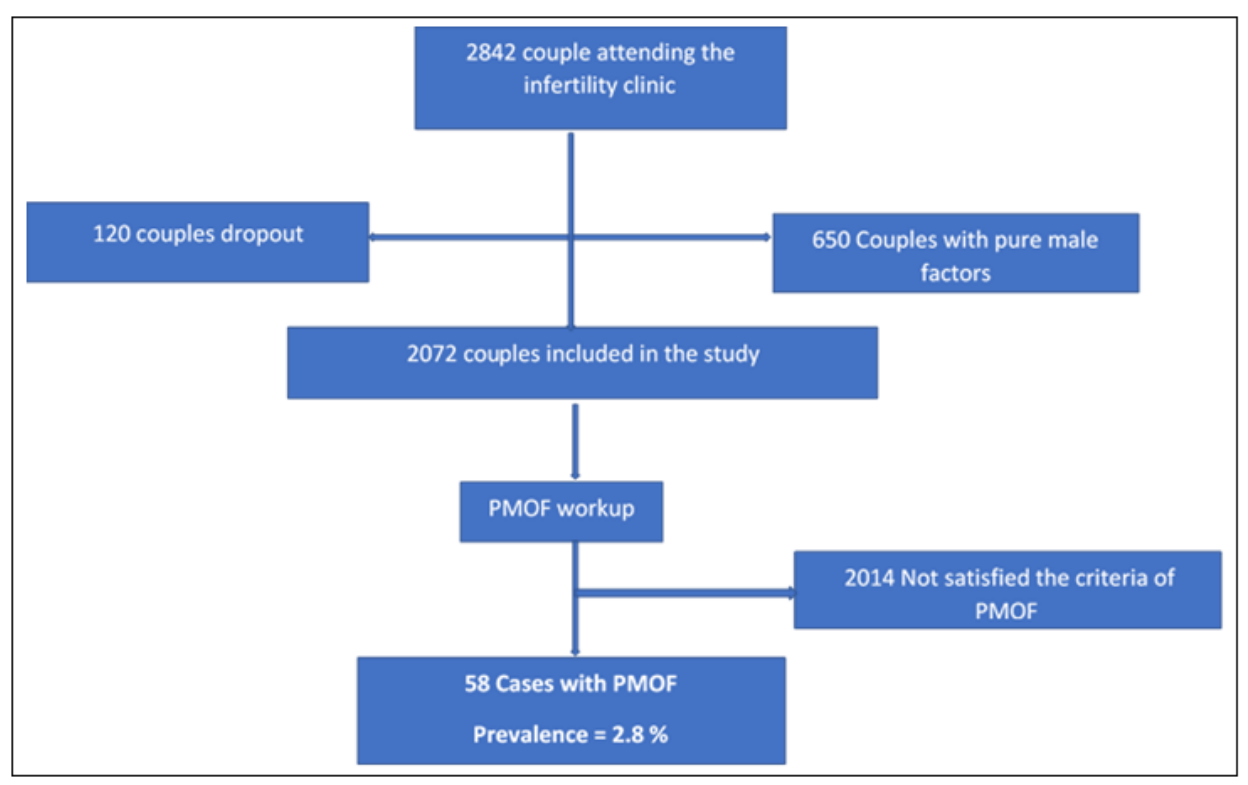

Fig-1: Patients' selection

Table-1: PMOF according to the age-group

\begin{tabular}{|l|l|}
\hline Age-group (years) & No. (\%) \\
\hline $20-30$ & $8(13.4 \%)$ \\
\hline $31-35$ & $18(30.5 \%)$ \\
\hline $36-40$ & $32(55.1 \%)$ \\
\hline
\end{tabular}

Table 2: Patients characteristics

\begin{tabular}{|lc|}
\hline \multicolumn{1}{|c|}{ Characteristics } & $\begin{array}{c}\text { Medium (range) } \\
\text { No. (percentage) }\end{array}$ \\
\hline Age at presentation (years) & $36(19-40)$ \\
BMI $\left(\mathrm{kg} / \mathrm{m}^{2}\right.$ ) & $30(21-40)$ \\
\hline Duration of infertility (years) & $4.5(1-14)$ \\
Type of infertility (n, \%) & \\
Primary & $49(84.4 \%)$ \\
Secondary & $9(15.5 \%)$ \\
\hline Duration of menstrual irregularity (years) & $7(1-16)$ \\
\hline Type of menstrual irregularity (n, \%) & \\
Oligomenorrhea & $35(60.5 \%)$ \\
Amenorrhea & $23(39.5 \%)$ \\
\hline Area of residence (n, \%) & \\
Urban & $32(55.2 \%)$ \\
suburban & $26(44.8 \%)$ \\
\hline Family history (n, \%) & $2(3.45 \%)$ \\
\hline Past surgical history (n, \%) & $14(24 \%)$ \\
Ovarian drilling & $1(1.7 \%)$ \\
Unilateral oophorectomy & $2(3.35 \%)$ \\
Ovarian cystectomy & $3(5.2 \%)$ \\
Lower abdominal or pelvic surgery & $8(13.8 \%)$ \\
\hline
\end{tabular}


Table-3: Hormonal level

\begin{tabular}{|l|l|}
\hline Hormone & Medium (range) \\
\hline FSH-1(IU/1) & $31(28-114)$ \\
\hline FSH-2 (IU/1) & $46(30-361)$ \\
\hline LH (IU/1) & $22(29-82)$ \\
\hline$E_{2}(\mathrm{ng} / \mathrm{ml})$ & $15(1-58)$ \\
\hline AMH $(\mathrm{ng} / \mathrm{ml})$ & $0.13(0.01-1.2)$ \\
\hline AFC & $1(0.00-5)$ \\
\hline
\end{tabular}

\section{DISCUSSION}

The prevalence of PMOF is very variable and ranged between 0.9 and $2 \%$ in most previously published studies [1, 3-5]. However, the data for these studies were collected from the general population. As infertility or low fertility are the most disturbing problem for these patients, this study aimed to evaluate the prevalence of PMOF among infertile women, and up to our knowledge, this study was the first study to assess the PMOF prevalence in the infertile subjects. The recorded prevalence of PMOF among infertile subjects in this study was $2.8 \%$ and $35 \%$ of the affected women were aged 35 years and below.

The median age of the included patients was 36 years and this was similar to previously recorded by another study where the mean age was 36.2 years [3]. The same study [3] reported a similar BMI $(28.86+6.62)$ as recorded by the present study.

Infertility was the main complaint of all the included participants as our center dealing with infertility. Most of the included women were having oligomenorrhea than amenorrhea in contrast to a previous study [19] where amenorrhea was the main presenting symptom.

$3.45 \%$ of the patient had a family history of the PMOF, this rate was lower than the rate of 4 to $31 \%$ reported by previous studies [20-22]. However, these studies gave the incidence among the postmenopausal and HRT registry whereas the incidence of the current study was taken from a younger age group, so by the time they reached the age of natural menopause a higher number of family members might be affected.

About 5-10\% of women with PMOF conceived spontaneously and this achievement of pregnancy could be due to the residual function of ovarian follicles [23]. In the current study; $3.45 \%$ of eligible subjects got spontaneous pregnant, but none resulted in live-birth.

Occult ovarian failure was defined as a triad of menstrual irregularity, infertility, and high FSH levels [24]. However, women with occult PMOF may not be recognized until they present with infertility problem [23]. In the present study, menstrual irregularity preceded infertility by a median duration of 2.5 years. Similarly, previous studies reported that menstrual irregularity predated infertility by a median duration of 1.8 and 2 years $[25,26]$ respectively, and this can be considered as a first marker in the natural history of the PMOF and fertility preservation might be attempted during this time.

The past obstetric outcome of included women was poor, only $15 \%$ of them were having live-birth before the onset of the problem and 17 women were having failed IUI and/or ICSI trails and this could reflect their poor ovarian reserve. The only solution presently available for the infertility problem in women with absent follicular reserve is ovum donation which is not allowed in our population because of religious reasons. So, early screening and fertility preservation by gonadal tissue and/or embryo freezing should be considered for women at risk of PMOF, in young women who require cancer treatments, or those who have a strong family history of PMOF.

PMOF not only affecting the woman's reproductive potential, but also associated with increased risk of the general and mental health of the affected women $[9,10,27]$. Therefore, they should be counseled about the use of hormonal replacement therapy and cardiovascular disease prevention, osteoporosis, and fracture risk reduction. The sexual dysfunction and the psychological impact of early menopause should also be considered and the women may benefit from referral to a mental health professional [28, 29].

Because of the associated long-term health consequences, the data to estimate the prevalence of PMOF in previously published studies was taken either from the general population or from the registry of hormone replacement therapy (HRT). However, as the lower fertility or even infertility are the most distressing complaints to every woman with PMOF and because there was no data concerning the actual PMOF prevalence available to date, among the Libyan population. This study aimed to assess the prevalence of PMOF among infertile women attending one public center in Albayda/Libya as this may reflect the real magnitude of the problem in the whole Libyan population. In an attempt to reduce the incidence of PMOF in the future, to preserve fertility, and to avoid the adverse effect on the women's general and mental health. 
Limitations; substantial under-diagnosis remains a probability because of the small size of the study cohort as the study was limited to women who seek infertility medical care in a single public center. Also, many cases were not included because of the lack of follow up to have a second FSH level measurement to fulfill the criteria for diagnosis of PMOF.

\section{CONCLUSION}

The prevalence of PMOF in this study was $2.8 \%$ indicating a higher prevalence of PMOF among infertile women than previously reported prevalence in the general population. This study also confirms the low reproductive potential of these women.

\section{REFERENCES}

1. Coulam, C. B., Adamson, S. C., \& Annegers, J. F. (1986). Incidence of premature ovarian failure. Obstetrics and gynecology, 67(4), 604-606.

2. ESHRE Guideline Group on POI, Webber, L., Davies, M., Anderson, R., Bartlett, J., Braat, D., ... \& Janse, F. (2016). ESHRE Guideline: management of women with premature ovarian insufficiency. Human Reproduction, 31(5), 926937.

3. Haller-Kikkatalo, K., Uibo, R., Kurg, A., \& Salumets, A. (2015). The prevalence and phenotypic characteristics of spontaneous premature ovarian failure: a general population registry-based study. Human reproduction, 30(5), 1229-1238.

4. Mishra, G. D., Pandeya, N., Dobson, A. J., Chung, H. F., Anderson, D., Kuh, D., ... \& Lee, J. S. (2017). Early menarche, nulliparity and the risk for premature and early natural menopause. Human Reproduction, 32(3), 679-686.

5. Lagergren, K., Hammar, M., Nedstrand, E., Bladh, M., \& Sydsjö, G. (2018). The prevalence of primary ovarian insufficiency in Sweden; a national register study. BMC women's health, 18(1), 175.

6. Persani, L., Rossetti, R., \& Cacciatore, C. (2010). Genes involved in human premature ovarian failure. Journal of molecular endocrinology, 45(5), 257.

7. Nelson, L. M. (2009). Primary ovarian insufficiency. New England Journal of Medicine, 360(6), 606-614.

8. Goswami, D., \& Conway, G. S. (2007). Premature ovarian failure. Hormone Research in Paediatrics, 68(4), 196-202.

9. Rahman, I., Åkesson, A., \& Wolk, A. (2015). Relationship between age at natural menopause and risk of heart failure. Menopause, 22(1), 12-16.

10. Gallagher, J. C. (2007). Effect of early menopause on bone mineral density and fractures. Menopause, 14(3), 567-571.

11. Tao, X. Y., Zuo, A. Z., Wang, J. Q., \& Tao, F. B. (2016). Effect of primary ovarian insufficiency and early natural menopause on mortality: a metaanalysis. Climacteric, 19(1), 27-36.

12. Atsma, F., Bartelink, M. L. E., Grobbee, D. E., \& van der Schouw, Y. T. (2006). Postmenopausal status and early menopause as independent risk factors for cardiovascular disease: a metaanalysis. Menopause, 13(2), 265-279.

13. Bakalov, V. K., Vanderhoof, V. H., Bondy, C. A., \& Nelson, L. M. (2002). Adrenal antibodies detect asymptomatic auto-immune adrenal insufficiency in young women with spontaneous premature ovarian failure. Human Reproduction, 17(8), 2096-2100.

14. Betterle, C., Rossi, A., Pria, S. D., Artifoni, A., Pedini, B., Gavasso, S., \& Caretto, A. (1993). Premature ovarian failure: autoimmunity and natural history. Clinical endocrinology, 39(1), 3543.

15. Orshan, S. A., Ventura, J. L., Covington, S. N., Vanderhoof, V. H., Troendle, J. F., \& Nelson, L. M. (2009). Women with spontaneous 46, XX primary ovarian insufficiency (hypergonadotropic hypogonadism) have lower perceived social support than control women. Fertility and sterility, 92(2), 688-693.

16. Schmidt, P. J., Luff, J. A., Haq, N. A., Vanderhoof, V. H., Koziol, D. E., Calis, K. A., ... \& Nelson, L. M. (2011). Depression in women with spontaneous 46, XX primary ovarian insufficiency. The Journal of Clinical Endocrinology \& Metabolism, 96(2), E278-E287.

17. Gleicher, N., Kim, A., Weghofer, A., \& Barad, D. H. (2012). Toward a better understanding of functional ovarian reserve: AMH (AMHo) and FSH (FSHo) hormone ratios per retrieved oocyte. The Journal of Clinical Endocrinology \& Metabolism, 97(3), 995-1004.

18. Ramalho de Carvalho, B., Gomes Sobrinho, D. B., Vieira, A. D. D., Resende, M. P. S., Barbosa, A. C. P., Silva, A. A., \& Nakagava, H. M. (2012). Ovarian reserve assessment for infertility investigation. ISRN obstetrics and gynecology, 2012.

19. Gleicher, N., Weghofer, A., \& Barad, D. H. (2011). Defining ovarian reserve to better understand ovarian aging. Reproductive biology and endocrinology, 9(1), 23.

20. Conway, G. S., Kaltsas, G., Patel, A., Davies, M. C., \& Jacobs, H. S. (1996). Characterization of idiopathic premature ovarian failure. Fertility and sterility, 65(2), 337-341.

21. Cramer, D. W., Xu, H., \& Harlow, B. L. (1995). Family history as a predictor of early menopause. Fertility and sterility, 64(4), 740-745.

22. Torgerson, D. J., Thomas, R. E., \& Reid, D. M. (1997). Mothers and daughters menopausal ages: is there a link?. European Journal of Obstetrics \& Gynecology and Reproductive Biology, 74(1), 63 66. 
23. Gleicher, N., Kushnir, V. A., \& Barad, D. H. (2015). Prospectively assessing risk for premature ovarian senescence in young females: a new paradigm. Reproductive Biology and Endocrinology, 13(1), 34.

24. Cameron, I. T., O'shea, F. C., Rolland, J. M., Hughes, E. G., Kretser, D. M. D., \& Healy, D. L. (1988). Occult ovarian failure: a syndrome of infertility, regular menses, and elevated folliclestimulating hormone concentrations. The Journal of Clinical Endocrinology \& Metabolism, 67(6), 1190-1194.

25. Izhar, R., Husain, S., Tahir, S., \& Husain, S. (2017). Occult form of premature ovarian insufficiency in women with infertility and oligomenorrhea as assessed by poor ovarian response criteria. Journal of reproduction \& infertility, 18(4), 361.
26. Shestakova, I. G., Radzinsky, V. E., \& Khamoshina, M. B. (2016). Occult form of premature ovarian insufficiency. Gynecological Endocrinology, 32(sup2), 30-32.

27. Atsma, F., Bartelink, M. L. E., Grobbee, D. E., \& van der Schouw, Y. T. (2006). Postmenopausal status and early menopause as independent risk factors for cardiovascular disease: a metaanalysis. Menopause, 13(2), 265-279.

28. Cox, L., \& Liu, J. H. (2014). Primary ovarian insufficiency: an update. International journal of women's health, 6, 235.

29. van der Stege, J. G., Groen, H., van Zadelhoff, S. J., Lambalk, C. B., Braat, D. D., van Kasteren, Y. M., ... \& Hoek, A. (2008). Decreased androgen concentrations and diminished general and sexual well-being in women with premature ovarian failure. Menopause, 15(1), 23-31. 